\title{
Finite-Volume Time-Domain (FVTD) Method and its Application to the Analysis of Hemispherical Dielectric-Resonator Antennas
}

\author{
Dirk Baumann, Christophe Fumeaux, Pascal Leuchtmann, Rüdiger Vahldieck \\ Swiss Federal Institute of Technology, ETHZ, IFH, 8092 Zürich, Switzerland
}

\begin{abstract}
In this paper the Finite-Volume Time-Domain (FVTD) method is refined and applied to analyze a probe-fed hemispherical dielectric resonator antenna (DRA). To improve the applicability of the FVTD method to microwave problems, a new scheme is introduced taking advantage of the method's inherent flux separation into incoming and outgoing waves. A 3D simulation is performed using an unstructured tetrahedral mesh permitting precise modeling of curved surfaces and fine structures. The obtained results are compared to those from other methods.
\end{abstract}

\section{INTRODUCTION}

The Finite-Volume Time-Domain (FVTD) method has been used since the late $80^{\prime}$ 's in computational electromagnetics $[1,2]$. This technique solves numerically Maxwell's curl equations by integration over small elementary volumes. Since there are no requirements on the shape of the elementary volumes, the method is naturally suited for use in unstructured meshes. Therefore, the FVTD method is a powerful alternative to the FiniteDifference Time-Domain (FDTD) method for a whole class of problems where conformal meshing is advantageous. The modeling of curved and oblique structures in the classical Yee FDTD grid involves stair-casing approximations that are very demanding on storage resources for accurate simulation. Existing modifications of the original Yee algorithm adapt the technique to irregular meshes at the cost of an increased complexity. On the contrary, conformal meshing is naturally taken into account in the FVTD algorithm. Practical FVTD meshes make use of small polyhedrons as elementary cells (finite volumes) with typical edge length in the order of $\lambda 10$. The meshes handled here are composed of tetrahedrons that can approximate curved surfaces very accurately with reasonable mesh fineness.

In the past the FVTD method has been applied predominantly for scattering problems, for example to determine radar cross-sections. Application of the technique in microwave engineering requires both the implementation of electromagnetic sources and the characterization of ports. By exploiting the algorithm's inherent flux separation in incoming and outgoing waves we introduced a new scheme for full-wave field excitation and for full-wave S-parameter extraction. These extensions make the FVTD method especially suited for microwave device simulations.

To demonstrate these novel features the FVTD algorithm is applied to a challenging problem: The analysis of probe-fed Dielectric resonator antenna (DRA) with hemispherical surface.

\section{THE FVTD ALGORITHM}

The FVTD technique is based on the conservative form of Maxwell's curl equations [3] integrated over the elementary polyhedral volume $V_{i}$ using the divergence theorem

$$
\begin{aligned}
-\frac{\partial}{\partial t} \iiint_{V_{i}} \vec{B} d v & =\oiint_{\partial V_{i}} \vec{n} \times \vec{E} d a=\sum_{k=1}^{N_{i}} \vec{n}_{k} \times \iint_{F_{k}} \vec{E} d a \\
\frac{\partial}{\partial t} \iiint_{V_{i}} \vec{D} d v & =\oiint_{\partial V_{i}} \vec{n} \times \vec{H} d a=\sum_{k=1}^{N_{i}} \vec{n}_{k} \times \iint_{F_{k}} \vec{H} d a
\end{aligned}
$$

In these equations, $\partial V_{i}$ represents the boundary of $V_{i}$ and is composed of $N_{i}$ planar faces with areas $F_{k}$ and outer normal unit vectors $\vec{n}_{k}$. The surface integrals are interpreted as "fluxes" through the cell faces. For numerical computations, the exact equations (1) are discretized as follows: The volume integrals on the lefthand side (LHS) are estimated using mean values of $\vec{B}$ and $\vec{D}$ in the volume $V_{i}$. On the right-hand side (RHS), the surface integrals are approximated using the surface mean values of $\vec{E}$ and $\vec{H}$ over each face of the polyhedron. The discretized equations in a tetrahedral cell $(N=4)$ are then written as:

$$
\begin{aligned}
-\frac{\partial}{\partial t}\langle\vec{B}\rangle_{V} & =\frac{1}{V} \sum_{k=1}^{4}\left(\vec{n}_{k} \times\langle\vec{E}\rangle_{F_{k}}\right) F_{k} \\
\frac{\partial}{\partial t}\langle\vec{D}\rangle_{V} & =\frac{1}{V} \sum_{k=1}^{4}\left(\vec{n}_{k} \times\langle\vec{H}\rangle_{F_{k}}\right) F_{k}
\end{aligned}
$$

where the \langle\rangle denotes spatial mean values. The coupled equations (2) represent the foundation of a cell-centered FVTD scheme. Inserting the material equations $\vec{D}=\varepsilon \vec{E}$ and $\vec{B}=\mu \vec{H}$ into (2), localizing mean volume values in 
the barycenter of the cells, and mean area values in the barycenter of the cell faces permit to write approximate coupled equations for the time-dependent $\vec{E}$ and $\vec{H}$. In this formulation, electric and magnetic field components are stored at the same locations (cell barycenters).

Explicit FVTD update equations are obtained following a chosen time-marching scheme. In general, the LHS of (2) will contain "old" and "new" values of the timedependent field functions, whereas the RHS will contain time-averaged field values. Independently of the timemarching scheme used, we can basically separate a FVTD time step into two stages as illustrated in Fig. 1:

1) Tangential field components on the faces of the cell are computed on the basis of field values at the barycenter of the considered cell and its direct neighbors.

2) The barycenter values are updated using fluxes through the cell faces according to (2) and following the chosen time-marching scheme.

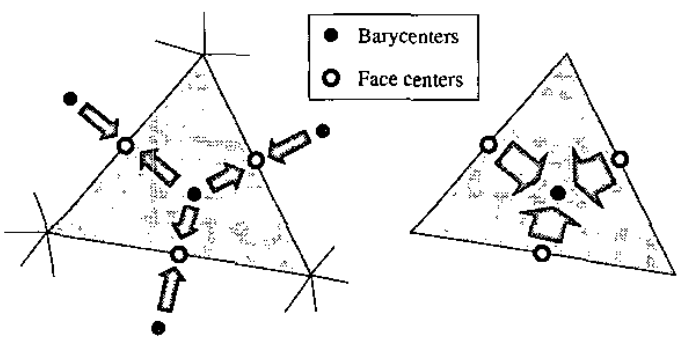

(a)

(b)

Fig. 1. 2D illustration of the two stages of one FVTD iteration step: (a) Tangential fields at face centers are computed from the values of the fields at barycenters, (b) Barycenter values are updated using approximated fluxes through the faces.

Different implementations of the FVTD method are distinguished by different approximations in the first stage. The commonly used schemes separate incoming and outgoing fluxes through the surfaces. For each face of a considered cell,

(a) outgoing fluxes of tangential fields are computed from barycenter values in the considered cell

(b) incoming fluxes of tangential fields are computed from barycenter values in the neighbor cell.

In the algorithm presented here, second-order accuracy in space is achieved by using estimated gradients in the cell barycenters according to the Monotonic Upwind Scheme for Conservation Laws (MUSCL) [3]. Secondorder accuracy in time is attained applying the LaxWendroff predictor-corrector scheme [3]. In the following some relevant aspects are listed.

\section{A. Mesh Generation}

The tetrahedral mesh generation is performed with a commercial program (Altair( ${ }^{\circledR}$ HyperMesh(B). The raw data from the mesher is transformed during a preprocessing step into the geometrical data necessary for the FVTD algorithm. Lists of nodes, triangular faces and tetrahedrons are stored in the computer memory, including geometrical characteristics and connectivity. The structure is built to minimize bookkeeping problems associated with the unstructured mesh during the FVTD iteration.

\section{B. Radiation Boundary Conditions (RBC)}

The Silver-Müller condition, a very simple and natural $\mathrm{RBC}$, is applied on the outer boundary of the computational domain. This RBC exploits the structure of the FVTD scheme and sets fluxes incoming from the outside of the computational domain to zero. Although this RBC is only exact for normal incidence on the boundary, good performances can be achieved by adapting the shape of the outer boundary to the problem. For scattering and antenna problems, a spherical outer boundary permits generally to satisfy the normal-incidence condition of radiated fields fairly well.

\section{Sources}

There are several practical ways of defining sources of electromagnetic (EM) waves in the simulated problem. One practical excitation scheme in FVTD is to impress the known field distribution of a propagating mode on a transverse plane of a transmission line (e.g. a coaxial cable). The triangulation of the excitation surface must be embedded in the tetrahedral mesh. From the numerical point of view, the energy is introduced in the FVTD computational domain as additional fluxes through the triangles that build the excitation surface (i.e. in stage 2 of the iteration). The scheme has a significant advantage: If placed on the outer boundary of the computational domain, a source plane provides EM excitation towards the inside, but also represents a Silver-Müller RBC toward the outside. In broadband applications, a Gaussian pulse is used as time function of the excitation.

\section{Scattering Parameters}

Ports are defined as a plane in the FVTD mesh, similar to source surfaces. To determine the S-parameters of a port, the separation of incoming and outgoing fluxes inherent to the FVTD algorithm is exploited. On the tetrahedron faces (triangles) that build the port, we are able to distinguish tangential fields associated to the two opposite directions of propagation through the port $\left(\vec{E}_{t}^{+}\right.$ vs. $\vec{E}_{t}^{-}$, and $\vec{H}_{t}^{+}$vs. $\left.\vec{H}_{i}^{-}\right)$. No extra computations are necessary to obtain these field components since they are used in the FVTD update equations. The application of a Discrete Fourier Transform (DFT) on the fly permits to retrieve the frequency dependence of these field 
components. The complex Poynting vectors in both directions are determined locally on each triangle and integrated over the port plane to calculate the incident and reflected power at the port. According to the definition of $S$-parameters, the square root of the power is used to calculate them.

\section{E. Memory Requirements}

The FVTD scheme applied in a tetrahedral mesh requires storage of geometrical data. For second-order accuracy storage of field components at three different time steps as well as field gradients is additionally necessary. In comparison, the classical FDTD with its regular grid and staggered formulation requires around 10 times less memory per cell. For complex 3D geometries however, the conformal FVTD meshing permits accurate simulations with much coarser grids [4]. Thus a significant saving of resources is achieved despite larger cost per cell.

\section{THE HEMISPHERICAL DRA}

Dielectric resonator antennas (DRA) are attractive as alternative to microstrip antennas because of their small size and large bandwidth [5]. The use of low-loss materials and the small influence of conductor losses permit to achieve high radiation efficiency. The coupling of power to the structure is easily achieved using coaxial probes, microstrip or coplanar transmission lines. Dielectric resonators of any shape may be used for antenna design but geometries like rectangles, cylinders, rings, and hemispheres are predominantly used. Typical DRA structures include large dielectric contrasts, curved surfaces and small feed dimensions. A probe-fed hemispherical DRA $[6,7]$ is simulated here to demonstrate the ability of the FVTD method to handle this type of problems and to prove the correct and accurate operation of the ports. The geometry is depicted in Fig. 2.

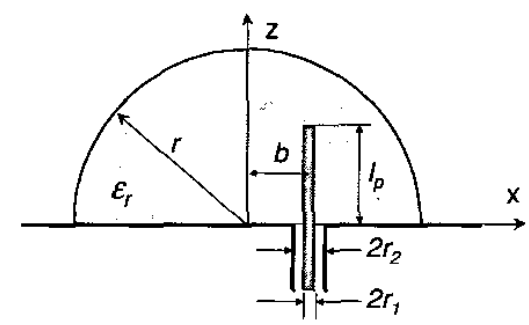

Fig. 2. Geometry of the probe-fed hemispherical DRA.

The results presented in the following are obtained for a hemisphere with radius $r=12.5 \mathrm{~mm}$ and a dielectric permittivity $\varepsilon_{r}=9.5$ similarly as in [7]. The feed structure for excitation of the resonant $\mathrm{TE}_{111}$ mode has the following geometrical characteristics: $b=6.5 \mathrm{~mm}$, $l_{p}=6.5 \mathrm{~mm}$. The coaxial cable has the inner and outer radii $r_{1}=0.63 \mathrm{~mm}, r_{2}=2.25 \mathrm{~mm}$ and a permittivity of its isolator $\varepsilon_{\text {coax }}=2.33$. The DRA is placed on a finite ground plane (circular plate with diameter $120 \mathrm{~mm}$ ). Figure 3 depicts the triangular mesh of the boundary surfaces of the device (the tetrahedral volume mesh is not shown in the representation). The picture permits to observe the large difference in cell densities between inside and outside meshes of the DRA dielectric. An even finer mesh is required for the precise modeling of the feed structure. The size of the tetrahedral cells adapts smoothly to the various discretization sizes of the enclosing surfaces. Small cells (compared to the dielectric wavelength of interest) will require small time steps for stability of the computation. However, the inhomogeneous meshes will avoid a 3D explosion of computational costs caused by fine geometrical structures or large dielectric contrasts.

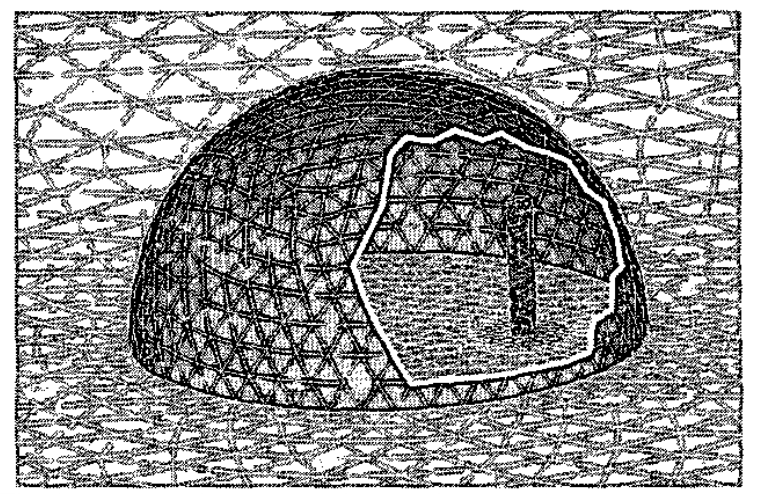

Fig. 3. Discretization of the hemispherical DRA. Only the skin triangulations of boundary surfaces are shown. The typical relative ratio of volumes between free-space cells and feed cells is $1000: 1$.

\section{RESULTS}

The resonance frequency corresponding to the fundamental $\mathrm{TE}_{111}$ mode of the structure is expected at around $3.6 \mathrm{GHz}$ [6]. The full $3-6 \mathrm{GHz}$ range is simulated in a single computational run using a Gaussian pulse with the appropriate bandwidth. The obtained return loss is represented in Fig. 4 and compared with results obtained by a commercial frequency-domain finite-element program (Ansoft HFSS ${ }^{\mathrm{TM}}$ ). The overall agreement between the two methods is fairly good. The discrepancy at larger frequencies may be explained by the coarser grids compared to the wavelengths. Figure 5 is a representation of the near-field distribution obtained with FVTD. This steady state was simulated using a sine-wave excitation near the fundamental mode $(f=3.6 \mathrm{GHz})$. The observation of the near-field distribution gives practical information on the excitation of a particular mode. 


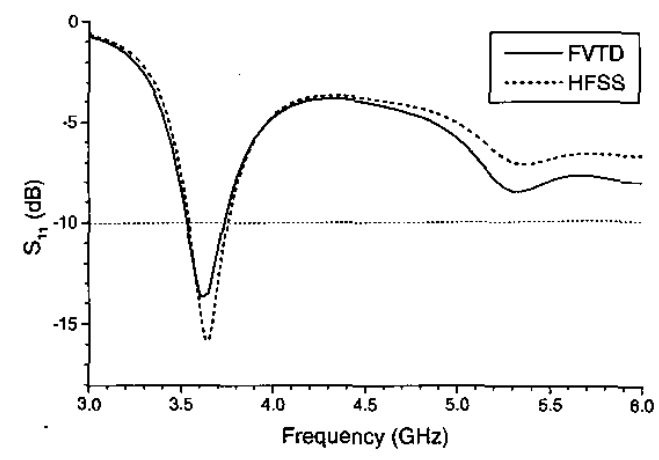

Fig. 4. Return loss of the hemispherical DRA computed with the FVTD method and compared with HFSS results.

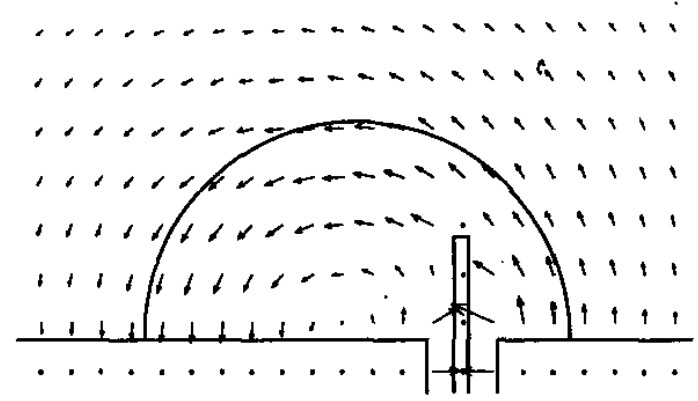

Fig. 5. Snapshot of the steady-state E-Field distribution in the E-plane at $3.6 \mathrm{GHz}$.

A good agreement between the far-field patterns computed with the FVTD method and those obtained with HFSS has been observed. Fig. 6 displays the radiation pattern close to the fundamental resonance with quasibroadside characteristics.

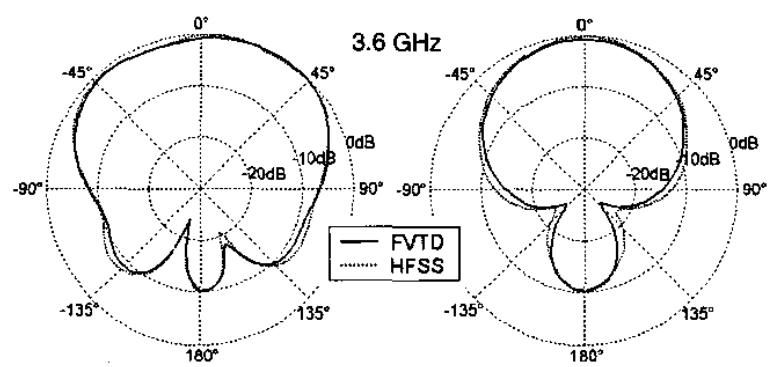

Fig. 6. Normalized radiation pattern of the DRA close to resonance (3.6 GHz). LHR: E-plane, RHS: H-plane.

Variation of the probe length $l_{p}$ permits to control the input impedance of the DRA. A length of $l_{p}=8 \mathrm{~mm}$ shows the best return loss for the fundamental mode (Fig. 7). The achieved dynamic range approaches $30 \mathrm{~dB}$, proving the accurate performance of our $S$-parameter extraction based on the separation of fluxes.

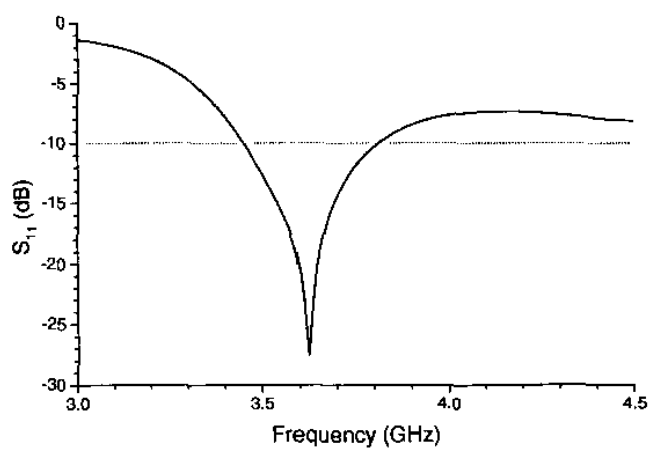

Fig. 7. Return loss of an impedance-matched DRA illustrating the dynamic range of FVTD ports. The probe length $l_{p}$ is $8 \mathrm{~mm}$.

\section{CONCLUSION}

The ability of the FVTD method for the modeling of complex microwave devices was demonstrated by analyzing a probe-fed hemispherical DRA. In a single simulation run, broadband near fields, radiation patterns and matching parameters are retrieved. The exploitation of flux-splitting allows an elegant and accurate definition of sources and ports. The method is universal and needs no changes for a broad range of antenna geometries.

The authors wish to acknowledge the assistance of Claudia Cibin for the HFSS simulations.

\section{REFERENCES}

[1] N.K. Madsen, R.W. Ziolkowski, "A three-dimensional modified finite volume technique for Maxwell's equations", Electromagnetics, vol. 10, pp. 147-161, 1990.

[2] V. Shankar, A.H. Mohammadian, W.F. Hall, "A timedomain, finite-volume treatment for the Maxwell equations", Electromagnetics, vol. 10, pp. 127-145, 1990.

[3] P. Bonnet, X. Ferrieres, B.L. Michielsen, P. Klotz, J.L. Roumiguières, "Finite-volume time domain method", Chapter 9 in "Time domain electromagnetics", ed. by S.M. Rao, Academic Press, San Diego, 1999.

[4] M. Yang, Y. Chen, R. Mittra, "Hybrid finite-difference/ finite-volume time-domain analysis for microwave integrated circuits with curved PEC surfaces using a nonuniform rectangular grid", IEEE Trans. Microwave Theory and Tech., vol. MTT-48, pp 969-975, June 2000.

[5] R.K. Mongia, P. Bhartia, "Dielectric resonator antennas - A review and general design relations for resonant frequency and bandwidth", Int. J. of Microwave and Millimeter-Wave Computer-Aided Engineering, vol. 4, pp. 230-247, 1994.

[6] K.W. Leung, K.M. Luk, K.Y.A. Lai, D. Lin, "Theory and experiment of a coaxial probe fed hemispherical dielectric resonator antenna", IEEE Trans. Antennas Propagation, vol. 41, pp. 1390-1398, Oct. 1993.

[7] K.W. Leung, K.K. Tse, K.M. Luk, E.K.N. Yung, "Crosspolarization characteristics of a probe-fed hemispherical dielectric resonator antenna", IEEE Trans, Antennas Propagation, vol. 47, pp. 1228-1230, July 1999. 